\title{
Rhytidectomy approach for surgical treatment of branchial cyst
}

\author{
Lorena Pingarrón Martin • Javier Arias Gallo • \\ Javier González Martín-Moro • \\ Edurne Palacios Weiss • Miguel Burgueño García
}

Published online: 1 December 2009

(C) Springer-Verlag 2009

\begin{abstract}
Surgical treatment of benign diseases of the neck produces a cervicotomy scar. A modified rhytidectomy incision has been used since 2003 in our department in selected cases of parotid gland tumours. However, there is no mention in bibliography about using facelift approach for treatment of cervical diseases. We have operated on three patients with brachial cysts using this technique. We introduce a clinical case of a 28-year-old woman with a right cervical swelling at the level of the anterior side of the sternocleidomastoid muscle. This technique allows a wide surgical approach. There is no donor-site morbidity, minimum additional operating time, hidden scar and no extra cost, and patients are very satisfied with the results.
\end{abstract}

Keywords Branchial cyst $\cdot$ Rhytidectomy approach

Surgical treatment of benign diseases of the neck produces a cervicotomy scar. Depending on the conditions of the patient (age, obesity or tendency to hypertrophic scars), results may be unsatisfactory.

L. Pingarrón Martin $(\bowtie) \cdot J$. Arias Gallo

J. González Martín-Moro • E. Palacios Weiss •

M. Burgueño García

La Paz University Hospital,

Madrid, Spain

e-mail: lorenapingarron@yahoo.es
A modified rhytidectomy incision has been used since 2003 in our department in selected cases of parotid gland tumours. A pretragal incision is continued around the auricular lobule, into the postauricular sulcus and up to the midportion of the mastoid process. From there, the incision is directed backwards and downwards following the postauricular hairline. Exposure afforded with this incision is wide enough for most of the benign tumours of the parotid gland.

However, there is no mention in bibliography about using the facelift approach for the surgical treatment of cervical diseases.

Devauchelle described a similar approach for mandibular reconstruction with microvascularized graft, avoiding unaesthetic cervical incisions [1].

Branchial cysts and fistulae are pathologies frequently found in young people in which a cervicotomy approach may be quite conspicuous. These pathologies are usually located high and lateral in the neck, making them suitable for the rhytidectomy approach [2].

We have operated on three patients with branchial cysts. The slight longer operating time is compensated by the complete camouflage of the scar. This technique allows a wide surgical approach.

There is no donor-site morbidity, minimum additional operating time, hidden scar and no extra cost, and patients are very satisfied with the results.

We introduce a clinical case of a 28 -year-old woman with a right cervical swelling located at the level of the anterior side of the sternocleidomastoid muscle. 


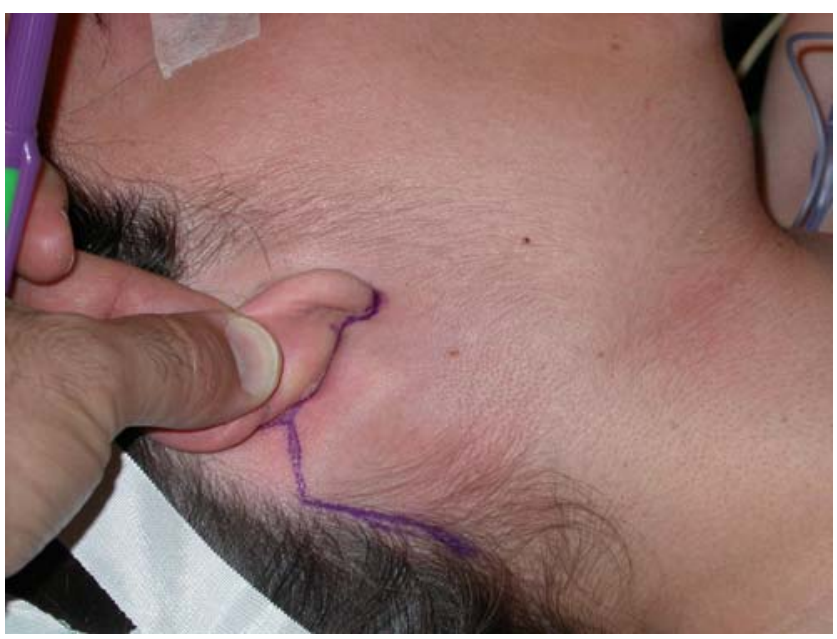

Fig. 1 Lesion with an incision around the auricular lobule and along the retroauricular line

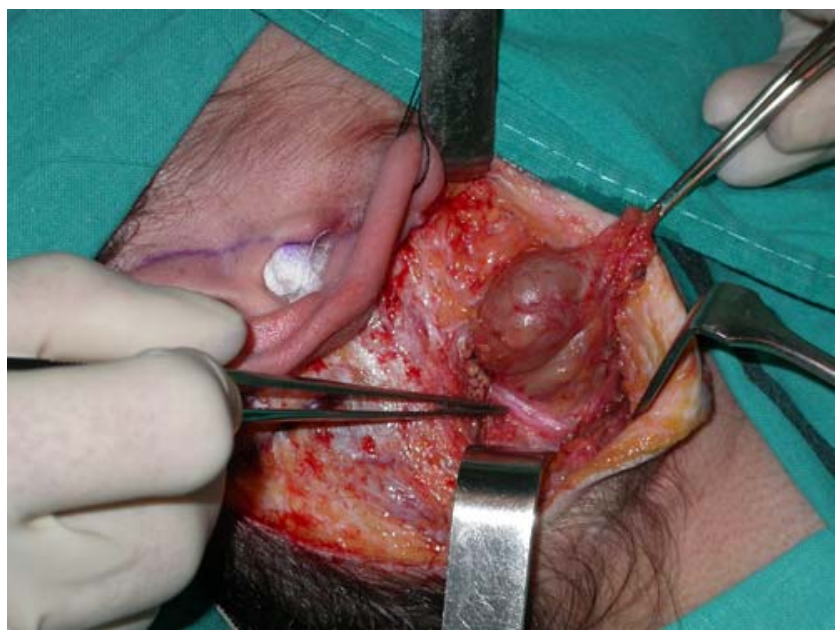

Fig. 2 The branchial cyst is dissected in the same way as when the transverse cervicotomy incision is performed

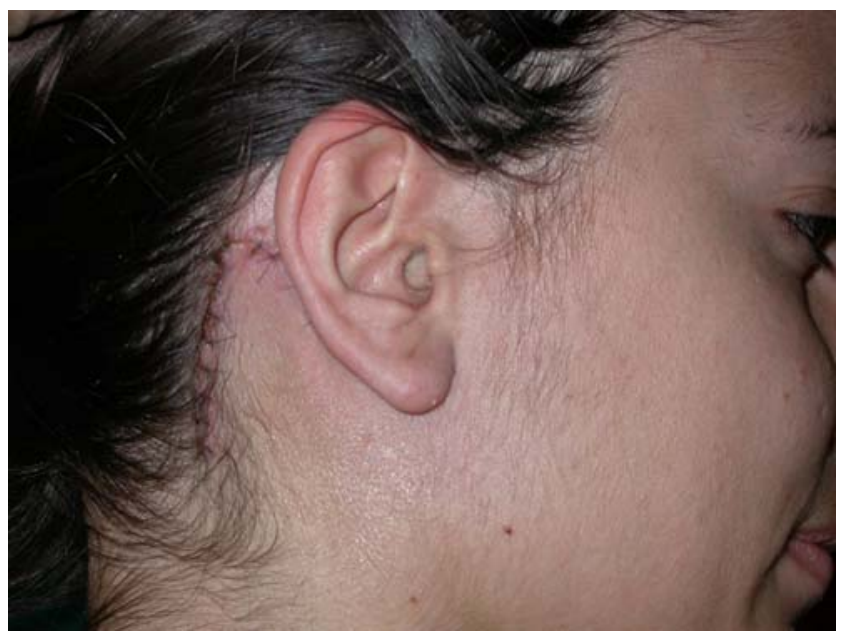

Fig. 3 Short- and long-time follow-up shows a very good aesthetic and functional (Fig. 3) results

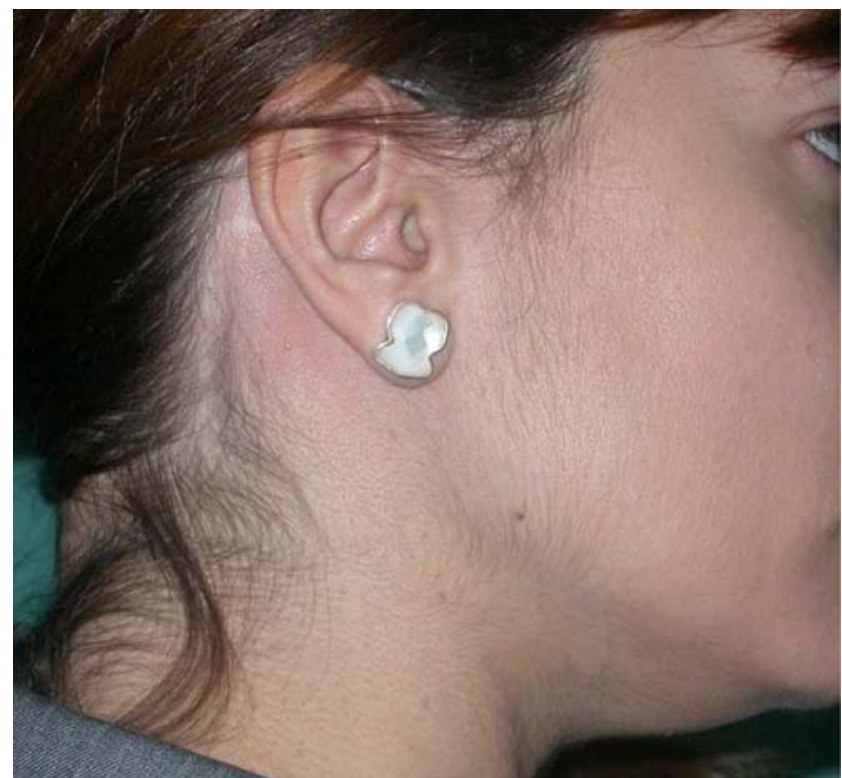

Fig. 4 One year follow-up postsurgery. It is noticed the completely unconspicuous scar

A previous fine needle aspitation of the lesion suggested a branchial cyst [3].

Under general anaesthesia, we approached the lesion with an incision around the auricular lobule and along the retroauricular line, with a 3-mm distance far away from the retroauricular line in extension to the hairline (Fig. 1).

This incision itself is wide enough to avoid any prolongation at the preauricular region. The branchial cyst is dissected in the same way as when the transverse cervicotomy incision is performed (Fig. 2), recommending using aspirative drenage for $24 \mathrm{~h}$.

We can conclude that short- and long-time follow-ups show a very good aesthetic and functional (Figs. 3 and 4) results.

Rhytidectomy approach is a suitable alternative for treatment of cervical lesions located in level II, especially in young patients to avoid anaesthetics scars.

\section{References}

1. Golledge J, Ellis H (1994) The aetiology of lateral cervical (branchial) cysts: past and present theories. J Laryngol Otol 108:653-659

2. Daoud FS (2005) Branchial cyst: an often forgotten diagnosis. Asian J Surg 28(3):174-178

3. Glosser JW, Pires CAS (2003) Branchial cleft or cervical lymphoepithelial cysts. J Am Dent Assoc 134(1):81-86 